\title{
AVES VULNERABLES A COLISIONAR CONTRA TORRES EÓLICAS EN RIVAS, NICARAGUA, ANTES DE SU CONSTRUCCIÓN
}

\section{VULNERABLE BIRDS TO COLLIDE AGAINST WIND TOWERS IN RIVAS, NICARAGUA, BEFORE THEIR CONSTRUCTION}

José Manuel Zolotoff Pallais ${ }^{1}$

(recibido/received: 30-marzo-2020; aceptado/accepted:21-mayo-2021)

RESUMEN: Se aplicó un Índice de Vulnerabilidad de Aves (IVA) y Mapa de Vulnerabilidad Potencial (MVP) para determinar cuáles son las especies de aves más susceptibles a colisionar con torres eólicas y los sitios con mayor riesgo, en una central eólica al sur de la ciudad de Rivas. Se colocaron transectos en dos zonas donde se colocarían las torres: Pastizales sin 'Árboles y Pastizales con Árboles. También se realizaron transectos en hábitats adyacentes como Costa del lago de Nicaragua y Bosque Ripario. El IVA se calculó con nueve factores (altura de vuelo, tipo de vuelo, longitud de ala, peso, estatus, abundancia, estado reproductivo, estado de conservación internacional y nacional). Se calculó el MVP total a partir de todas las especies detectadas, y MVP medio solo utilizando las especies que superaron la mediana del IVA específico. El riesgo de colisión por hábitat se calculó determinando que menor al percentil 50 se considera de riesgo bajo, y de riesgo alto cuando el percentil sea mayor que 50. Los valores más altos de vulnerabilidad se encuentran en las especies: Rabihorcado Magno (Fregata magnificens), el Zopilote Negro (Coragyps atratus), el Zopilote Cabecirroja (Cathartes aura), Águila Pescadora (Pandion haliaetus), Caracara Crestado (Caracara cheriway), Zanate Nicaragüense (Quiscalus nicaraguensis), y la Garza Grande (Ardea herodias). El bosque ripario y pastizales con árboles son los sitios con mayor riesgo de colisión para instalar torres eólicas. El IVA y MVP constituyen herramientas importantes que permiten identificar los riesgos potenciales de colisión de aves en centrales eólicas antes de su construcción.

PALABRAS CLAVES: Aerogenerador, evaluación de impacto ambiental, índice de vulnerabilidad, riesgo de colisión.

ABSTRACT: A Bird Vulnerability Index (BVI) and Potential Vulnerability Map (PVM) was applied to determine which are the most susceptible bird species to collide with wind towers and the riskiest sites, in a wind power plant south from the city of Rivas. Transects were placed in two areas where the towers would be placed: Grasslands without Trees and Grasslands with Trees. Transects were also made in adjacent habitats such as Lake Nicaragua Coast and Riparian Forest. The BVI was calculated with nine factors (Flight Height, Type of Flight, Wingspan, Weight,

\footnotetext{
${ }^{1}$ Responsable de Investigación y Capacitación Reserva Natural Volcán Mombacho. Fundación Cocibolca. Nicaragua.josezolotoff@gmail.com
} 
José M. Zolotoff $P$.

Status, Abundance, Reproductive Status, International and National Conservation Status). The total PVM was calculated from all detected species, and average PMV only using species that exceeded the specific BVI median. The risk of habitat collision was calculated by determining that less of 50th percentile is considered to be low risk, and high risk when the percentile is greater than 50. The highest vulnerability index is found in the species: Magnificent Frigatebird (Fregata magnificens), Black Vulture (Coragyps atratus), Turkey Vulture (Cathartes aura), Osprey (Pandion haliaetus), Crested Caracara (Caracara cheriway), Nicaraguan Grackle (Quiscalus nicaraguensis), and Great Heron (Ardea herodias). The riparian forest and grassland with trees are the sites with the highest risk of collision to install wind towers. The BVI and PVM are important tools that allow the identification of potential risks of bird collision with wind towers before their construction.

KEYWORDS: Collision risk, environmental impact assessment, vulnerability index, wind generator.

\section{INTRODUCCIÓN}

La energía eólica es uno de los sectores con más crecimiento en la industria energética. Este tipo de energía tiene el potencial de reducir impactos al ambiente causados por el uso de combustibles fósiles para generar energía convencional. Sin embargo, la preocupación del impacto de esta energía "verde" sobre los ecosistemas, en especial, sobre las colisiones de aves y murciélagos con este tipo de infraestructura sigue siendo una preocupación a tener en cuenta en la etapa previa a su construcción.

Según la American Wind Wildlife Institute (AWWI, 2016), la capacidad de la energía eólica para generar electricidad sin emisiones de carbono se espera que reduzca el riesgo de efectos potencialmente catastróficos para la vida silvestre ante un cambio climático inminente. Sin embargo, los impactos adversos de las instalaciones de energía eólica a la vida silvestre han sido documentados, particularmente en las aves y murciélagos (Arnett et al., 2008; Strickland et al., 2011; AWWI, 2016).

Nicaragua comenzó el aprovechamiento de energía eólica en el año 2007, cuando se instalaron treinta aerogeneradores en la finca Amayo, en Rivas. Otros proyectos en funcionamiento son Eolo de Nicaragua S.A, Blue Power Energy S.A y Alba Vientos, siendo inminente el auge que están teniendo los parques eólicos en Nicaragua.

El istmo de Rivas es un área importante de migración de aves, sobre todo de rapaces actuando como un cuello de botella. McCrary \& Young (2008) describen la migración de aves rapaces cerca del empalme La Virgen, en Rivas, a más de 19,000 aves de 10 especies de rapaces volando entre los meses de agosto y noviembre; así como a más de 50,000 individuos de Golondrina Común (Hirundo rustica), Avión Púrpura (Progne subis), Golondrina Bicolor (Tachycineta bicolor), Avión Zapador (Riparia riparia), Golondrina Gorginegra (Hirundo pyrrhonota) y Golondrina Alirrasposa 
José M. Zolotoff $P$.

Norteña (Stelgidopteryx serripennis), entre otros grupos de aves migratorias como acuáticas y paseriformes.

La utilidad de la presente investigación, se basa en proporcionar un Índice de Vulnerabilidad de Aves, que servirá para predecir los posibles impactos de especies de aves en torres eólicas, agregando un Mapa de Vulnerabilidad Potencial de la instalación de torres, con el fin de obtener información más precisa sobre los lugares de menor riesgo para la instalación de torres.

El método que se presenta en este artículo, es una herramienta de evaluación más detallada de los posibles impactos que puedan tener las centrales eólicas en la etapa de pre construcción sobre aves, y de esta forma tomar medidas correctivas desde el inicio, con vistas a reducir posibles colisiones durante la etapa de operación.

\section{METODOLOGÍA}

El estudio se realizó en la zona del proyecto Eolo de Nicaragua S.A. a $125 \mathrm{~km}$ de Managua a 13 $\mathrm{km}$ al sur de la ciudad de Rivas, Departamento de Rivas, en los meses de septiembre a octubre del 2011 (8 días) durante la migración de aves de otoño de norte a sur, y de marzo a abril del 2012 (8 días) durante la migración de aves de primavera de sur a norte, totalizando 16 días de monitoreo.

El proyecto posee 22 torres eólicas (instaladas en el 2014) con una longitud de torre de $85 \mathrm{~m}$ y una longitud $45 \mathrm{~m}$ por cada una de las tres aspas, con una capacidad de generación de $2.0 \mathrm{MW}$ cada uno, para una capacidad instalada total de $80.0 \mathrm{MW}$.

El estudio es de tipo observacional, ya que los datos fueron colectados de la fuente primaria; es longitudinal ya que recaba datos en diferentes puntos del tiempo realizando inferencias sobre el problema tratado, en este caso, el estudio abarca dos períodos específicos de tiempo en el cual se obtuvieron los datos, y de campo pues las mediciones se realizaron in situ (HernándezSampieri, Collado y Baptista, 2014).

Se colocaron transectos de ancho variable (Ralph et al., 1996; Wunderle, 1994), con un promedio de $1 \mathrm{~km}$ de longitud en cada uno de los cuatro tipos de hábitats presentes en la zona: Bosque Ripario (BR), Pastos sin Árboles (PSA), Pastos con Árboles (PCA) y Costa del Lago (CL). Dichos transectos se colocaron aproximadamente sobre la línea donde se contemplaba la instalación de torres eólicas en esa época. Si bien no se contempla la instalación de torres sobre la línea de Costa del Lago y Bosque Ripario, se incluyeron estos hábitats con fines comparativos y para documentar la riqueza y abundancia de especies (Figura 1).

El Higo Revista Científica / Volumen 11. No. 01, pp. 69-89/ junio 2021 
José M. Zolotoff $P$.

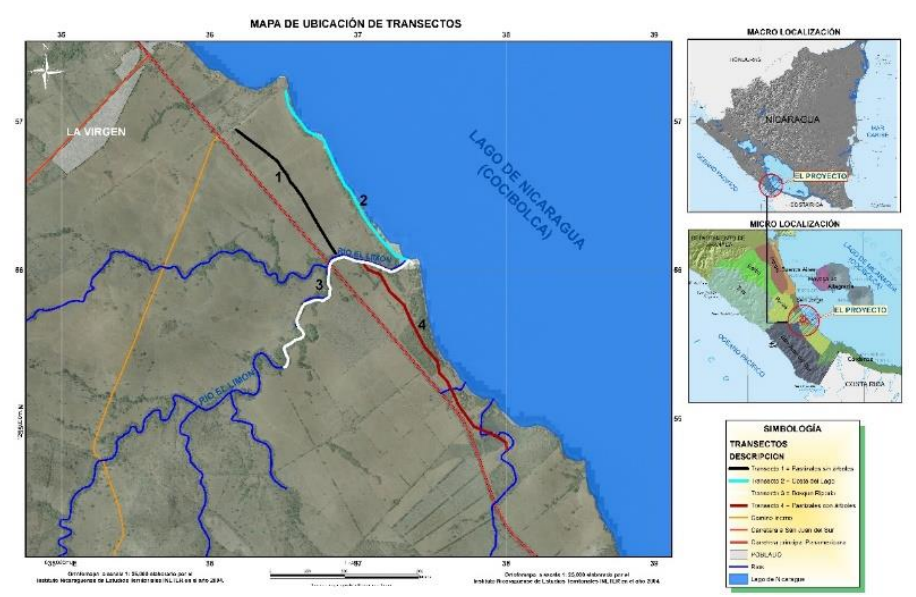

Figura 1.- Ubicación de transectos. Central eólica Eolo de Nicaragua S.A 2011-2012.

Se realizaron dos monitoreos por día por cada uno de los hábitats de forma simultánea iniciando el primero a las 6:00 y culminando a las 9:30 horas y el segundo de 15:00 a 17:30 horas.

Para cada individuo de las especies de aves migratorias y residentes observados en cada hábitat se tomaron los siguientes datos en los diferentes transectos: especie, abundancia, altura de vuelo, dirección de vuelo, actividad, distancia del ave al sendero y al observador.

El Índice de Vulnerabilidad de Aves (IVA) se obtuvo para cada especie detectada y en cada uno de los diferentes tipos de hábitats, seleccionaron nueve factores valorados en una escala que va de uno (menor vulnerabilidad) hasta cuatro (mayor vulnerabilidad) agrupados en dos grupos, aquellos factores que tienen que ver con el riesgo de colisión y los relacionados con la sensibilidad de las especies para un total de nueve factores. Los promedios de ambos grupos se multiplican para obtener el IVA de cada especie por hábitat.

La ecuación (1) muestra el cálculo del IVA:



Donde:

A: Altura de vuelo de las aves. Tomada según la altura de vuelo de cada ave en relación a la altura de la torre y longitud de aspa. La ponderación se realizó como: 1: > $145 \mathrm{~m}, 2$ : 0-25 m, 3: 26-40 m y 130-145 m, 4: 40-125 m. 
José M. Zolotoff $P$.

B: Tipo de vuelo. Basada en los datos tomado en campo para cada ave en cada tipo de hábitat monitoreado. La ponderación se realizó como: 1: Ave posada, 2: Forrajeando, 3: Cazando y volando, 4: Planeando

C: Longitud alar. Es la extensión máxima de las alas de punta a punta. Aves con longitudes grandes de alas pueden ser más propensas a colisionar por tener más área de contacto. La información se ha tomado de la base de datos de Cornell Lab of Ornithology (2015) para cada especie de ave, realizando una tabla de intervalos de frecuencia. La ponderación se realizó como: 1. 0-65 cm., 2. $65-117 \mathrm{~cm}, 3.117-168 \mathrm{~cm}, 4 .>168 \mathrm{~cm}$.

D: Peso. Aves pesadas pueden ser más propensas a colisionar por tener menos maniobrabilidad. La información fue tomada de Styles \& Skutch (1989) para cada especie de aves, realizando una tabla de intervalos de frecuencia. La ponderación se realizó como: 1. 0-629 g, 2. 629-1252 g, 3. 1252-1876 g, 4. >1876 g.

E: Estatus. El tiempo que pasan las aves expuestas al peligro puede ser mayor en especies que residen todo el año en la zona de peligro. La información fue tomada de Martínez-Sánchez et al., (2014). La ponderación se realizó como: 1. Especie de Paso, 2. Especie Migratoria, 3. Especie Residente y Migratoria, 4. Especie Residente.

F: Abundancia. Se determinó la abundancia relativa presentada de forma cualitativa reconociendo cuatro categorías: Muy abundante, Abundante, Escaso y Raro, de forma tal que para aves se puede construir para cada hábitat a partir de la especie más abundante, definiendo el punto numérico de referencia al dividir entre las cuatro categorías de abundancia (Pérez, 2004). La ponderación se realizó como: 1. Raro, 2. Escaso, 3. Abundante, 4. Muy abundante.

G. Estado reproductivo. Este factor hace mención sobre la cantidad de huevos o puestas de cada especie. Especies con menos posturas pueden ser las más vulnerables por tener menos descendencia. La información fue tomada de Styles \& Skutch (1989) y de la base de datos de Cornell Lab of Ornithology (2015). La ponderación se realizó como: 1. >4 huevos, 2. 3-4 huevos, 3. 2 huevos, 4. 1 huevo.

H. Estado de conservación internacional (UICN). Para este factor se utilizó los criterios de la International Union for Conservation of Nature (IUCN, 2016). La ponderación se realizó como: 1. Baja preocupación, 2. Casi Amenazada, 3. Vulnerable, 4. En Peligro.

I. Estado de conservación nacional. Se utilizó los criterios de la lista roja de especies amenazadas en Nicaragua (Manzanarez et al., 2018). La ponderación se realizó como: 1. LC: Baja preocupación, 2. NT: Casi amenazado, 3. VU: Vulnerable, 4. EN: En peligro y En peligro crítico.

El Mapa de Vulnerabilidad Potencial (MVP) está dada por la ecuación (2):

El Higo Revista Científica / Volumen 11. No. 01, pp. 69-89/ junio 2021 
$\sum_{l}^{n} \ln \left(p_{i}+1\right) x I V A_{i}$

Donde:

$p_{i}=$ Número de observaciones de la especie i en cada hábitat.

$I V A_{i}=$ Valor IVA de la especie $\mathrm{i}$.

$\mathrm{n}=$ Número de hábitats considerados para el análisis.

Se calculó el MVP total a partir de todas las especies detectadas, y MVP medio solo utilizando las especies que superaron la mediana del IVA específico, este último con el propósito de reducir la posibilidad de dilución de la vulnerabilidad por especies menos sensibles. El riesgo de colisión por hábitat se calculó por medio de percentiles, determinando que menor al percentil $50(<P 50)$ se considera de riesgo bajo, y de riesgo alto cuando el percentil sea mayor que 50 (>P50).

El resultado final de esta fórmula es una zonificación en los cuatro (4) tipos de hábitats seleccionados Bosque Ripario (BR), Pastos sin Árboles (PSA), Pastos con Árboles (PCA) y Costa del Lago $(\mathrm{CL})$ con diferentes niveles de riesgos por colisión, determinando qué sector o hábitats presenta mayor riesgo de colisión a la hora de la instalación de las torres eólicas.

\section{RESULTADOS Y DISCUSIÓN}

Composición de especies: Se identificaron 73 especies de aves lo que corresponde al $10 \%$ del total de especies para Nicaragua (Martínez-Sánchez et al., 2014), pertenecientes a 30 familias en 42,976 observaciones. Del total de especies, 49 son especies residentes en Nicaragua, 14 especies migratorias, tres especies de paso y siete especies con poblaciones residentes y migratorias. La familia con mayor porcentaje de especies fue la de las garzas (Ardeidae) con el $15 \%$, seguido de zanates, chichiltotes, tordos entre otros (Icteridae) con el 12\% (Tabla 1).

Tabla 1.- Abundancia de individuos en los diferentes hábitats. Eolo de Nicaragua S.A. 20112012.

\begin{tabular}{|l|l|c|c|c|c|c|c|}
\hline \multicolumn{1}{|c|}{ Nombre científico } & \multicolumn{1}{c|}{$\begin{array}{c}\text { Nombre común en } \\
\text { español }\end{array}$} & BR & CL & PCA & PSA & $\begin{array}{c}\text { Frecuencia } \\
\text { general }\end{array}$ & Porcentaje \\
\hline Actitis macularius & Andarríos Maculado & 9 & 36 & 0 & 0 & 45 & 1.5 \\
\hline Agamia agami & Garza Pechicastaña & 0 & 1 & 0 & 0 & 1 & 0.0 \\
\hline Agelaius phoeniceus & Tordo Sargento & 122 & 40 & 46 & 1 & 209 & 6.8 \\
\hline Amazilia rutila & Amazilia Canela & 0 & 0 & 2 & 0 & 2 & 0.1 \\
\hline Ardea alba & Garzón Grande & 24 & 23 & 10 & 1 & 58 & 1.9 \\
\hline Ardea herodias & Garzón Azul & 0 & 3 & 0 & 0 & 3 & 0.1 \\
\hline Brotogeris jugularis & Chocoyo Barbinaranja & 0 & 0 & 2 & 0 & 2 & 0.1 \\
\hline
\end{tabular}

El Higo Revista Científica / Volumen 11. No. 01, pp. 69-89/ junio 2021 


\begin{tabular}{|c|c|c|c|c|c|c|c|}
\hline Nombre científico & $\begin{array}{l}\text { Nombre común en } \\
\text { español }\end{array}$ & BR & CL & PCA & PSA & $\begin{array}{c}\text { Frecuencia } \\
\text { general }\end{array}$ & Porcentaje \\
\hline Bubulcus ibis & Garcilla Bueyera & 45 & 3 & 174 & 135 & 357 & 11.6 \\
\hline Buteo nitidus & Gavilán Gris & 0 & 0 & 2 & 0 & 2 & 0.1 \\
\hline Butorides virescens & Garcilla Capiverde & 17 & 6 & 0 & 0 & 23 & 0.7 \\
\hline Cairina moschata & Pato Real & 0 & 0 & 0 & 2 & 2 & 0.1 \\
\hline \begin{tabular}{|l} 
Calidris pusilla \\
\end{tabular} & $\begin{array}{l}\text { Correlimos } \\
\text { Semipalmeado }\end{array}$ & 0 & 1 & 0 & 0 & 1 & 0.0 \\
\hline Calocitta formosa & Urraca Copetona & 15 & 4 & 5 & 29 & 53 & 1.7 \\
\hline $\begin{array}{l}\text { Campylorhynchus } \\
\text { rufinucha }\end{array}$ & Saltapiñuela Nuquirrufa & 1 & 4 & 12 & 3 & 20 & 0.6 \\
\hline Caracara cheriway & \begin{tabular}{|l} 
Caracara Crestado \\
\end{tabular} & 7 & 46 & 62 & 19 & 134 & 4.3 \\
\hline Cathartes aura & Zopilote Cabecirrojo & 29 & 4 & 78 & 3 & 114 & 3.7 \\
\hline Charadrius collaris & Chorlitejo Collarejo & 0 & 1 & 0 & 0 & 1 & 0.0 \\
\hline Chloroceryle americana & Martín Pescador Verde & 2 & 0 & 3 & 0 & 5 & 0.2 \\
\hline Cochlearius & Pico Cuchara & 1 & 0 & 0 & 0 & 1 & 0.0 \\
\hline Columbina inca & Tortolita Colilarga & 1 & 20 & 8 & 1 & 30 & 1.0 \\
\hline Columbina talpacoti & Tortolita Rojiza & 1 & 0 & 0 & 6 & 7 & 0.2 \\
\hline Coragyps atratus & Zopilote Negro & 42 & 87 & 251 & 26 & 406 & 13.1 \\
\hline Crotophaga sulcirostris & Garrapatero Común & 72 & 29 & 3 & 28 & 132 & 4.3 \\
\hline $\begin{array}{l}\text { Dendrocygna } \\
\text { autumnalis }\end{array}$ & Piche Piquirojo & 2 & 5 & 32 & 0 & 39 & 1.3 \\
\hline Dives dives & Cacique Piquinegro & 1 & 0 & 0 & 0 & 1 & 0.0 \\
\hline Egretta caerulea & Garceta Azul & 4 & 0 & 0 & 2 & 6 & 0.2 \\
\hline Egretta thula & Garceta Patiamarilla & 18 & 10 & 0 & 5 & 33 & 1.1 \\
\hline Egretta tricolor & Garceta Tricolor & 1 & 3 & 0 & 0 & 4 & 0.1 \\
\hline Eupsittula canicularis & Perico Frentinaranja & 12 & 0 & 2 & 49 & 63 & 2.0 \\
\hline Falco peregrinus & Halcón Peregrino & 0 & 0 & 1 & 0 & 1 & 0.0 \\
\hline Falco sparverius & Cernícalo Americano & 0 & 0 & 0 & 1 & 1 & 0.0 \\
\hline Fregata magnificens & Rabihorcado Magno & 0 & 1 & 7 & 1 & 9 & 0.3 \\
\hline Himantopus mexicanus & Cigüeñuela Cuellinegra & 10 & 0 & 0 & 0 & 10 & 0.3 \\
\hline Icterus galbula & Chichiltote Norteño & 14 & 0 & 17 & 0 & 31 & 1.0 \\
\hline Icterus pustulatus & |Chichiltote Dorsilistado & 0 & 0 & 4 & 0 & 4 & 0.1 \\
\hline
\end{tabular}




\begin{tabular}{|c|c|c|c|c|c|c|c|}
\hline Nombre científico & $\begin{array}{c}\text { Nombre común en } \\
\text { español }\end{array}$ & BR & CL & PCA & PSA & $\begin{array}{c}\text { Frecuencia } \\
\text { general }\end{array}$ & Porcentaje \\
\hline Icterus spurius & Chichiltote Castaño & 0 & 0 & 1 & 0 & 1 & 0.0 \\
\hline Jacana spinosa & Jacana Centroamericana & 3 & 7 & 4 & 0 & 14 & 0.5 \\
\hline Megaceryle torquata & Martín Pescador Collarejo & 12 & 0 & 3 & 0 & 15 & 0.5 \\
\hline Melanerpes hoffmannii & Carpintero Nuquigualdo & 4 & 4 & 10 & 2 & 20 & 0.6 \\
\hline Mycteria americana & Cigüeña Americana & 10 & 0 & 1 & 0 & 11 & 0.4 \\
\hline Myiarchus tyrannulus & Güis Crestipardo Mayor & 1 & 8 & 2 & 0 & 11 & 0.4 \\
\hline Myiozetetes similis & Güis Chico & 3 & 0 & 1 & 4 & 8 & 0.3 \\
\hline Nyctanassa violacea & Martinete Cangrejero & 1 & 0 & 0 & 0 & 1 & 0.0 \\
\hline Oreothlypis peregrina & Reinita Verduzca & 3 & 0 & 0 & 0 & 3 & 0.1 \\
\hline Pandion haliaetus & Águila Pescadora & 2 & 7 & 1 & 1 & 11 & 0.4 \\
\hline Parabuteo unicinctus & Gavilán Charreteado & 4 & 10 & 37 & 11 & 62 & 2.0 \\
\hline $\begin{array}{l}\text { Parkesia } \\
\text { noveboracensis }\end{array}$ & Reinita Acuática Norteña & 9 & 1 & 10 & 0 & 20 & 0.6 \\
\hline Patagioenas flavirostris & Paloma Piquirroja & 2 & 0 & 1 & 2 & 5 & 0.2 \\
\hline Peucaea ruficauda & Sabanero Cabecilistado & 0 & 2 & 3 & 0 & 5 & 0.2 \\
\hline $\begin{array}{l}\text { Phalacrocorax } \\
\text { brasilianus }\end{array}$ & Cormorán Neotropical & 9 & 92 & 0 & 0 & 101 & 3.3 \\
\hline Piaya cayana & Cuco Ardilla & 0 & 0 & 1 & 0 & 1 & 0.0 \\
\hline Pitangus sulphuratus & Güis Común & 15 & 12 & 21 & 7 & 55 & 1.8 \\
\hline Protonotaria citrea & Reinita Cebecidorada & 1 & 0 & 0 & 0 & 1 & 0.0 \\
\hline Psarocolius montezuma & Oropéndola Mayor & 3 & 0 & 11 & 0 & 14 & 0.5 \\
\hline Quiscalus mexicanus & Zanate Grande & 16 & 144 & 119 & 225 & 504 & 16.3 \\
\hline Quiscalus nicaraguensis & Zanate Nicaragüense & 91 & 0 & 0 & 0 & 91 & 2.9 \\
\hline Rupornis magnirostris & Gavilán Chapulinero & 0 & 0 & 1 & 0 & 1 & 0.0 \\
\hline Setophaga petechia & Reinita Amarilla & 37 & 19 & 38 & 5 & 99 & 3.2 \\
\hline Sturnella magna & Zacatero Común & 5 & 1 & 0 & 27 & 33 & 1.1 \\
\hline Tachybaptus dominicus & Zampullín Enano & 2 & 0 & 0 & 0 & 2 & 0.1 \\
\hline Thalasseus maximus & Pagaza Real & 0 & 82 & 2 & 0 & 84 & 2.7 \\
\hline Thraupis episcopus & Tángara Azulada & 1 & 0 & 24 & 0 & 25 & 0.8 \\
\hline $\begin{array}{l}\text { Thryophilus } \\
\text { pleurostictus }\end{array}$ & Charralero Fajeado & 3 & 0 & 4 & 0 & 7 & 0.2 \\
\hline
\end{tabular}




\begin{tabular}{|l|l|c|c|c|c|c|c|}
\hline \multicolumn{1}{|c|}{ Nombre científico } & \multicolumn{1}{c|}{$\begin{array}{c}\text { Nombre común en } \\
\text { español }\end{array}$} & BR & CL & PCA & PSA & $\begin{array}{c}\text { Frecuencia } \\
\text { general }\end{array}$ & Porcentaje \\
\hline Tigrisoma mexicanum & Garza Tigre Gorgilisa & 2 & 0 & 1 & 0 & 3 & 0.1 \\
\hline Turdus grayi & Sensontle Pardo & 1 & 0 & 0 & 0 & 1 & 0.0 \\
\hline Tyrannus forficatus & Tijereta Rosada & 22 & 2 & 2 & 0 & 26 & 0.8 \\
\hline Tyrannus melancholicus & Tirano Tropical & 21 & 0 & 13 & 0 & 34 & 1.1 \\
\hline Tyrannus tyrannus & Tirano Norteño & 0 & 0 & 3 & 0 & 3 & 0.1 \\
\hline Vanellus chilensis & Avefría Sureña & 0 & 1 & 0 & 0 & 1 & 0.0 \\
\hline Zenaida asiatica & Tórtola Aliblanca & 2 & 0 & 2 & 1 & 5 & 0.2 \\
\hline Total general & & $\mathbf{6 8 9}$ & $\mathbf{7 1 6}$ & $\mathbf{1 0 1 7}$ & $\mathbf{5 9 6}$ & $\mathbf{3 0 8 8}$ & $\mathbf{1 0 0}$ \\
\hline
\end{tabular}

Clave: Bosque ripario (BR), Costa del lago (CL), Pastizales con árboles (PCA), Pastizales sin árboles (PSA)

La Tabla 2 muestra que el hábitat con mayor número de especies observadas fue el bosque ripario con 51 especies, seguido de los pastizales con árboles con 46 especies y el de mayor concentración de individuos.

Tabla 2.- Riqueza y abundancia de especies por tipo de hábitat. Eolo de Nicaragua S.A 20112012.

\begin{tabular}{|c|c|c|c|c|}
\hline Hábitat & BR & CL & PCA & PSA \\
\hline Especies & 51 & 35 & 46 & 27 \\
\hline Individuos & 735 & 719 & 1037 & 597 \\
\hline
\end{tabular}

Clave: Bosque ripario (BR), Costa del lago (CL), Pastizales con árboles (PCA), Pastizales sin árboles (PSA)

Análisis del Índice de Vulnerabilidad de Aves: Para el bosque ripario el mayor valor del IVA se presenta en el Zopilote Cabecirrojo (IVA= 5) (residente y migratorio), el Águila Pescadora (IVA= 5) (migratorio), El Zopilote Negro (IVA=4.7) y Querque (IVA=4.4). Para el caso del Zopilote, puede ser vulnerable a las colisiones durante la fase de operación del proyecto ya que pasa mucho tiempo sobrevolando el área. Las torres en las cercanías de este tipo de hábitat podrían ser un riesgo para las aves. El valor promedio del IVA para este hábitat es de 2.9 y la mediana para las especies en este hábitat es de 2.8 (Tabla 3).

Tabla 3.- Valores IVA y MVP paras las especies en bosque ripario. Eolo de Nicaragua S.A. 2011-2012.

\begin{tabular}{|l|l|c|c|c|}
\hline Nombre Científico & \multicolumn{1}{|c|}{ Nombre Común } & IVA & MVP TOTAL & MVP Med \\
\hline Cathartes aura & Zopilote Cabecirrojo & 5.0 & 17.00598691 & 17.00598691 \\
\hline Pandion haliaetus & Águila Pescadora & 5.0 & 5.493061443 & 5.493061443 \\
\hline
\end{tabular}

El Higo Revista Científica / Volumen 11. No. 01, pp. 69-89/ junio 2021 
José M. Zolotoff $P$.

\begin{tabular}{|c|c|c|c|c|}
\hline Nombre Científico & Nombre Común & IVA & MVP TOTAL & MVP Med \\
\hline Coragyps atratus & Zopilote Negro & 4.7 & 17.76122277 & 17.76122277 \\
\hline Caracara cheriway & Caracara Crestado & 4.4 & 9.241962407 & 9.241962407 \\
\hline $\begin{array}{l}\text { Quiscalus } \\
\text { nicaraguensis }\end{array}$ & Zanate Nicaragüense & 4.4 & 20.09683812 & 20.09683812 \\
\hline Mycteria americana & Cigüeña Americana & 4.2 & 9.991230303 & 9.991230303 \\
\hline Darabuteo unicinctus & Gavilán Charreteado & 4.2 & 6.705991302 & 6.705991302 \\
\hline $\begin{array}{l}\text { Patagioenas } \\
\text { flavirostris }\end{array}$ & Paloma Piquirroja & 4.0 & 4.394449155 & 4.394449155 \\
\hline Zenaida asiatica & Tórtola Aliblanca & 4.0 & 4.394449155 & 4.394449155 \\
\hline Riparia riparia & Avión Zapador & 4.0 & 30.64422553 & 30.64422553 \\
\hline Hirundo rustica & Golondrina Común & 4.0 & 29.87861669 & 29.87861669 \\
\hline $\begin{array}{l}\text { Psarocolius } \\
\text { montezuma }\end{array}$ & Oropéndo & 3.9 & 5.391144738 & 5.391144738 \\
\hline Tigrisoma mexicanum & Garza Tigre Gorgilisa & 3.9 & 4.272381123 & 4.272381123 \\
\hline Eupsittula canicularis & Perico Frentinaranja & 3.7 & .404814311 & 9.404814311 \\
\hline Butorides virescens & Garcilla Capiverde & 3.6 & 0.43745357 & 10.43745357 \\
\hline $\begin{array}{l}\text { Dendrocygna } \\
\text { autumnalis }\end{array}$ & Piche Piquirojo & 3.6 & 3.967211042 & 3.967211042 \\
\hline Agelaius phoeniceus & Tordo Sargento & 3.3 & 16.04061452 & 16.04061452 \\
\hline Bubulcus ibis & Garcilla Bueyera & 3.3 & 12.76213799 & 12.76213799 \\
\hline Melanerpes hoffmannii & \begin{tabular}{|l} 
Carpintero \\
Nuquigualdo
\end{tabular} & 3.3 & 5.364793041 & 5.364793041 \\
\hline $\begin{array}{l}\text { Cochlearius } \\
\text { cochlearius }\end{array}$ & Pico Cuchara & 3.1 & 2.117949718 & 2.117949718 \\
\hline Thraupis episcopus & Tángara Azulada & 3.1 & 2.117949718 & 2.117949718 \\
\hline Ardea alba & Garzón Grande & 2.9 & 9.298974605 & 9.298974605 \\
\hline Columbina inca & Tortolita Colilarga & 2.8 & 1.925408835 & 1.925408835 \\
\hline Columbina talpacoti & Tortolita Rojiza & 2.8 & 1.925408835 & 1.925408835 \\
\hline Egretta caerulea & Garceta Azul & 2.8 & 4.470660868 & 4.470660868 \\
\hline Myiarchus & $\begin{array}{l}\text { Güis Crestipardo } \\
\text { Mayor }\end{array}$ & 2.8 & 1.925408835 & 1.925408835 \\
\hline
\end{tabular}

El Higo Revista Científica / Volumen 11. No. 01, pp. 69-89/ junio 2021 
José M. Zolotoff $P$.

\begin{tabular}{|c|c|c|c|c|}
\hline Nombre Científico & Nombre Común & IVA & MVP TOTAL & MVP Med \\
\hline Quiscalus mexicanus & Zanate Grande & 2.8 & 12.56052383 & 12.56052383 \\
\hline Turdus grayi & Sensontle Pardo & 2.8 & 1.925408835 & 1.925408835 \\
\hline $\begin{array}{l}\text { Tyrannus } \\
\text { melancholicus }\end{array}$ & Tirano Tropical & 2.8 & 8.586229037 & 8.586229037 \\
\hline Calocitta formosa & Urraca Copetona & 2.7 & 7.393569926 & 270.1025072 \\
\hline Egretta thula & Garceta Patiamarilla & 2.7 & 7.851837278 & \\
\hline $\begin{array}{l}\text { Parkesia } \\
\text { noveboracensis }\end{array}$ & $\begin{array}{l}\text { Reinita Acuática } \\
\text { Norteña }\end{array}$ & 2.7 & 6.140226915 & \\
\hline $\begin{array}{l}\text { Phalacrocorax } \\
\text { brasilianus }\end{array}$ & Cormorán Neotropical & 2.7 & 6.140226915 & \\
\hline $\begin{array}{l}\text { Crotophaga } \\
\text { sulcirostris }\end{array}$ & Garrapatero Común & 2.4 & 10.48778975 & \\
\hline Nyctanassa violacea & Martinete Cangrejero & 2.4 & 1.694359775 & \\
\hline Setophaga petechia & Reinita Amarilla & 2.3 & 8.487701039 & \\
\hline $\begin{array}{l}\text { Campylorhynchus } \\
\text { rufinucha }\end{array}$ & $\begin{array}{l}\text { Saltapiñuela } \\
\text { Nuquirrufa }\end{array}$ & 2.2 & 1.540327068 & \\
\hline $\begin{array}{l}\text { Chloroceryle } \\
\text { americana }\end{array}$ & $\begin{array}{l}\text { Martín Pescador } \\
\text { Verde }\end{array}$ & 2.2 & 2.441360641 & \\
\hline Dives dives & Cacique Piquinegro & 2.2 & 1.540327068 & \\
\hline Egretta tricolor & Garceta Tricolor & 2.2 & 1.540327068 & \\
\hline Megaceryle torquata & $\begin{array}{l}\text { Martín Pescador } \\
\text { Collarejo }\end{array}$ & 2.2 & 5.699887461 & \\
\hline Myiozetetes similis & Güis Chico & 2.2 & 3.080654136 & \\
\hline Pitangus sulphuratus & Güis Común & 2.2 & 6.161308272 & \\
\hline $\begin{array}{l}\text { Tachybaptus } \\
\text { dominicus }\end{array}$ & Zampullín Enano & 2.2 & 2.441360641 & \\
\hline $\begin{array}{l}\text { Thryophilus } \\
\text { pleurostictus }\end{array}$ & Charralero Fajeado & 2.2 & 3.080654136 & \\
\hline Oreothlypis peregrina & Reinita Verduzca & 1.8 & 2.464523309 & \\
\hline Protonotaria citrea & Reinita Cebecidorada & 1.8 & 1.232261654 & \\
\hline
\end{tabular}

El Higo Revista Científica / Volumen 11. No. 01, pp. 69-89/ junio 2021 
José M. Zolotoff $P$.

\begin{tabular}{|l|l|c|l|l|}
\hline Nombre Científico & \multicolumn{1}{|c|}{ Nombre Común } & IVA & MVP TOTAL & MVP Med \\
\hline $\begin{array}{l}\text { Himantopus } \\
\text { mexicanus }\end{array}$ & $\begin{array}{l}\text { Cigüeñuela } \\
\text { Cuellinegra }\end{array}$ & 1.7 & 3.996492121 & \\
\hline Jacana spinosa & $\begin{array}{l}\text { Jacana } \\
\text { Centroamericana }\end{array}$ & 1.7 & 2.310490602 & \\
\hline Actitis macularius & Andarríos Maculado & 1.6 & 3.581799034 & \\
\hline Icterus galbula & Chichiltote Norteño & 1.6 & 4.212522535 & \\
\hline Tyrannus forficatus & Tijereta Rosada & 1.6 & 4.877435447 & \\
\hline Total & & & 368.49995 & \\
\hline
\end{tabular}

El bosque ripario es el único "refugio verde" que se encuentra en la zona, donde los animales pasan la noche, se alimentan y se protegen del sol en un mar de tierras deforestadas producto de una ganadería y agricultura extensiva con muchos años de historia en la zona, por lo que resulta importante la conservación de este tipo de bosque.

Para la costa del lago, las especies con mayor valor de vulnerabilidad es el Rabihorcado Magno (IVA= 5.7), un ave marina que se interna tierra adentro y que vuela a alturas más allá de los 200 $m$ de altura. El Zopilote Negro $($ IVA $=5)$ es otra especie vulnerable en esta zona ya que se le observa en las costas comiendo de los desperdicios que arroja el lago. Con el mismo valor de vulnerabilidad en este hábitat es el Águila Pescadora (IVA=5), la cual utiliza al lago de Nicaragua para cazar peces. El Zopilote Cabecirrojo (IVA=4.7) aparece también con valores altos a la mediana en este hábitat junto a la Garza Pechicastaña (IVA=4.3). Es importante señalar la presencia de más de cinco mil golondrinas en esta zona, especialmente de la golondrina Avión Zapador (IVA= 4.2) (especie migratoria de paso), así como la Golondrina Común (IVA= 3.6) (migratoria de paso), especies con valor de vulnerabilidad por encima de la mediana, forrajean la línea costera del lago en busca de chayules muy frecuentes en esta zona. El valor promedio del IVA para este hábitat es de 3.2 y la mediana es de 3.1 (Tabla 4).

Tabla 4.- Valores IVA y MVP paras las especies en costa del lago. Eolo de Nicaragua S.A. 2011-2012.

\begin{tabular}{|l|l|c|l|l|}
\hline \multicolumn{1}{|c|}{ Nombre Científico } & \multicolumn{1}{|c|}{$\begin{array}{c}\text { Nombre Común en } \\
\text { español }\end{array}$} & IVA & MVP total & MVP Med \\
\hline Fregata magnificens & Rabihorcado Magno & 5.7 & 3.92783402 & 3.92783402 \\
\hline Coragyps atratus & Zopilote Negro & 5.0 & 22.3866841 & 22.3866841 \\
\hline Pandion haliaetus & Águila Pescadora & 5.0 & 10.3972077 & 10.3972077 \\
\hline Cathartes aura & Zopilote Cabecirrojo & 4.7 & 7.60012348 & 7.60012348 \\
\hline Agamia agami & Garza Pechicastaña & 4.3 & 2.96512961 & 2.96512961 \\
\hline
\end{tabular}

El Higo Revista Científica / Volumen 11. No. 01, pp. 69-89/ junio 2021 
José $M$. Zolotoff $P$.

\begin{tabular}{|c|c|c|c|c|}
\hline Nombre Científico & $\begin{array}{c}\text { Nombre Común en } \\
\text { español }\end{array}$ & IVA & MVP total & MVP Med \\
\hline Riparia riparia & Avión Zapador & 4.2 & 29.0251533 & 29.0251533 \\
\hline Caracara cheriway & Caracara Crestado & 4.2 & 16.0422817 & 16.0422817 \\
\hline Quiscalus mexicanus & Zanate Grande & 4.2 & 20.7363906 & 20.7363906 \\
\hline Dendrocygna autumnalis & Piche Piquirojo & 3.9 & 6.96795349 & 6.96795349 \\
\hline Ardea herodias & Garzón Azul & 3.6 & 5.00606297 & 5.00606297 \\
\hline Butorides virescens & Garcilla Capiverde & 3.6 & 7.02689776 & 7.02689776 \\
\hline Hirundo rustica & Golondrina Común & 3.6 & 30.4014151 & 30.4014151 \\
\hline Columbina inca & Tortolita Colilarga & 3.3 & 10.1484081 & 10.1484081 \\
\hline Parabuteo unicinctus & Gavilán Charreteado & 3.3 & 7.99298424 & 7.99298424 \\
\hline Sturnella magna & Zacatero Común & 3.3 & 2.3104906 & 2.3104906 \\
\hline Thalasseus maximus & Pagaza Real & 3.3 & 14.7294687 & 14.7294687 \\
\hline Ardea alba & Garzón Grande & 3.3 & 10.5935128 & 10.5935128 \\
\hline Bubulcus ibis & Garcilla Bueyera & 3.1 & 4.23589944 & 4.23589944 \\
\hline Melanerpes hoffmannii & Carpintero Nuquigualdo & 3.1 & 4.91772695 & 4.91772695 \\
\hline Tyrannus forficatus & Tijereta Rosada & 3.1 & 3.35687088 & 3.35687088 \\
\hline Parkesia noveboracensis & Reinita Acuática Norteña & 3.0 & $\mid 2.07944154$ & 220.768495 \\
\hline Phalacrocorax brasilianus & Cormorán Neotropical & 2.9 & 13.0941763 & \\
\hline Agelaius phoeniceus & Tordo Sargento & 2.8 & $\mid 10.315478$ & \\
\hline Peucaea ruficauda & Sabanero Cabecilistado & 2.8 & 3.0517008 & \\
\hline Myiarchus tyrannulus & Güis Crestipardo Mayor & 2.8 & 6.1034016 & \\
\hline Calocitta formosa & Urraca Copetona & 2.7 & 4.29183443 & \\
\hline Campylorhynchus rufinucha & Saltapiñuela Nuquirrufa & 2.7 & 4.29183443 & \\
\hline Crotophaga sulcirostris & Garrapatero Común & 2.7 & 9.06985968 & \\
\hline Egretta tricolor & Garceta Tricolor & 2.7 & 3.69678496 & \\
\hline Pitangus sulphuratus & Güis Común & 2.7 & 6.83986495 & \\
\hline Setophaga petechia & Reinita Amarilla & 2.3 & 6.99004197 & \\
\hline Egretta thula & Garceta Patiamarilla & 2.2 & 5.32865616 & \\
\hline Actitis macularius & Andarríos Maculado & 2.0 & 7.22183583 & \\
\hline Vanellus chilensis & Avefría Sureña & 2.0 & 1.38629436 & \\
\hline Jacana spinosa & Jacana Centroamericana & 1.8 & 3.81230949 & \\
\hline
\end{tabular}

El Higo Revista Científica / Volumen 11. No. 01, pp. 69-89/ junio 2021 
José M. Zolotoff $P$.

\begin{tabular}{|l|l|c|l|l|}
\hline \multicolumn{1}{|c|}{ Nombre Científico } & \multicolumn{1}{|c|}{$\begin{array}{c}\text { Nombre Común en } \\
\text { español }\end{array}$} & IVA & MVP total & MVP Med \\
\hline Charadrius collaris & Chorlitejo Collarejo & 1.7 & 1.1552453 & \\
\hline Calidris pusilla & $\begin{array}{l}\text { Correlimos } \\
\text { Semipalmeado }\end{array}$ & 1.6 & 1.07822895 & \\
\hline Total & & $\mathbf{3 1 0 . 5 7 5 4 8 4}$ & \\
\hline
\end{tabular}

Las especies con valores de vulnerabilidad alto en los pastizales con árboles lo constituyen el Rabihorcado Magno (IVA=6.3), seguido del Zopilote Negro (IVA= 5.8). En esta zona existen lugares inundados por lo que se cuenta con la presencia del Garzón Grande (IVA=5.3) y de la Cigüeña Americana (IVA= 5), seguido de las especies Garzón Grande y Zopilote Cabecirrojo con un IVA de 5 respectivamente. El valor promedio del IVA para este hábitat es de 3.2 y la mediana es de 3.1 (Tabla 5).

Tabla 5.- Valores IVA y MVP paras las especies en pastizal con árboles. Eolo de Nicaragua S.A. 2011-2012.

\begin{tabular}{|l|l|c|c|c|}
\hline \multicolumn{1}{|c|}{ Nombre Científico } & $\begin{array}{c}\text { Nombre común en } \\
\text { español }\end{array}$ & IVA & MVP total & MVP Med \\
\hline Fregata magnificens & Rabihorcado Magno & 6.3 & 13.1697964 & 13.1697964 \\
\hline Coragyps atratus & Zopilote Negro & 5.8 & 32.255003 & 32.255003 \\
\hline Ardea alba & Garzón Grande & 5.3 & 12.7887748 & 12.7887748 \\
\hline Cathartes aura & Zopilote Cabecirrojo & 5.3 & 23.0609748 & 23.0609748 \\
\hline Mycteria americana & Cigüeña Americana & 5.0 & 3.4657359 & 3.4657359 \\
\hline Bubulcus ibis & Garcilla Bueyera & 4.4 & 22.9546043 & 22.9546043 \\
\hline Parabuteo unicinctus & Gavilán Charreteado & 4.4 & 16.1670496 & 16.1670496 \\
\hline Riparia riparia & Avión Zapador & 4.2 & 29.9564897 & 29.9564897 \\
\hline Caracara cheryway & Caracara Crestado & 4.2 & 17.2630614 & 17.2630614 \\
\hline Pandion haliaetus & Águila Pescadora & 4.2 & 2.88811325 & 2.88811325 \\
\hline Hirundo rustica & Golondrina Común & 3.9 & 31.809271 & 31.809271 \\
\hline Buteo nitidus & Gavilán Gris & 3.9 & 4.27238112 & 4.27238112 \\
\hline Dendrocygna autumnalis & Piche Piquirojo & 3.9 & 13.5975294 & 13.5975294 \\
\hline Tigrisoma mexicanum & Garza Tigre Gorgilisa & 3.9 & 2.69557237 & 2.69557237 \\
\hline Tachycineta albilinea & Golondrina Lomiblanca & 3.8 & 15.920688 & 15.920688 \\
\hline Eupsittula canicularis & Perico Frentinaranja & 3.7 & 4.02824506 & 4.02824506 \\
\hline
\end{tabular}


José $M$. Zolotoff $P$.

\begin{tabular}{|c|c|c|c|c|}
\hline Nombre Científico & $\begin{array}{c}\text { Nombre común en } \\
\text { español }\end{array}$ & IVA & MVP total & MVP Med \\
\hline Parkesia noveboracensis & $\begin{array}{l}\text { Reinita Acuática } \\
\text { Norteña }\end{array}$ & 3.7 & 8.79228267 & 8.79228267 \\
\hline Patagioenas flavirostris & Paloma Piquirroja & 3.7 & 2.54153966 & 2.54153966 \\
\hline Zenaida asiatica & Tórtola Aliblanca & 3.7 & 4.02824506 & 4.02824506 \\
\hline Rupornis magnirostris & Gavilán Chapulinero & 3.6 & 2.50303148 & 2.503031485 \\
\hline Psarocolius montezuma & Oropéndola Mayor & 3.6 & 8.97327401 & 8.97327401 \\
\hline Agelaius phoeniceus & Tordo Sargento & 3.3 & 12.8338253 & 12.8338253 \\
\hline Melanerpes hoffmannii & Carpintero Nuquigualdo & 3.3 & 7.99298424 & 7.99298424 \\
\hline Piaya cayana & Cuco Ardilla & 3.3 & 2.3104906 & 2.3104906 \\
\hline Falco peregrinus & Halcón Peregrino & 3.1 & 2.11794972 & 2.11794972 \\
\hline Quiscalus mexicanus & Zanate Grande & 3.1 & $\mid 14.628447$ & 14.628447 \\
\hline Setophaga petechia & Reinita Amarilla & 3.0 & 10.9906849 & 313.01536 \\
\hline Peucaea ruficauda & Sabanero Cabecilistado & 2.8 & 3.85081767 & \\
\hline Amazilia rutila & Amazilia Canela & 2.8 & 3.0517008 & \\
\hline Columbina inca & Tortolita Colilarga & 2.8 & 6.1034016 & \\
\hline Myiarchus tyrannulus & Güis Crestipardo Mayor & 2.8 & 3.0517008 & \\
\hline Myiozetetes similis & Güis Chico & 2.8 & 1.92540883 & \\
\hline Tyrannus melancholicus & Tirano Tropical & 2.8 & 7.3307148 & \\
\hline Brotogeris jugularis & Chocoyo Barbinaranja & 2.7 & 2.92963277 & \\
\hline Crotophaga sulcirostris & Garrapatero Común & 2.7 & 3.69678496 & \\
\hline Calocitta formosa & Urraca Copetona & 2.4 & 4.37985648 & \\
\hline Megaceryle torquatus & Megaceryle torquatus & 2.4 & 3.38871955 & \\
\hline Campylorhynchus rufinucha & Saltapiñuela Nuquirrufa & 2.2 & 5.69988746 & \\
\hline Chloroceryle americana & Martín Pescador Verde & 2.2 & 3.08065414 & \\
\hline Icterus pustulatus & Chichiltote Dorsilistado & 2.2 & 3.57652869 & \\
\hline Pitangus sulphuratus & Güis Común & 2.2 & 6.86898323 & \\
\hline Thalasseus maximus & Pagaza Real & 2.2 & 2.44136064 & \\
\hline Thryophilus pleurostictus & Charralero Fajeado & 2.2 & 3.57652869 & \\
\hline Tyrannus tyrannus & Tirano Norteño & 2.2 & 3.08065414 & \\
\hline
\end{tabular}


José M. Zolotoff $P$.

\begin{tabular}{|l|l|c|l|l|}
\hline \multicolumn{1}{|c|}{ Nombre Científico } & \multicolumn{1}{|c|}{$\begin{array}{c}\text { Nombre común en } \\
\text { español }\end{array}$} & IVA & MVP total & MVP Med \\
\hline Jacana spinosa & $\begin{array}{l}\text { Jacana } \\
\text { Centroamericana }\end{array}$ & 1.7 & 2.68239652 & \\
\hline Thraupis episcopus & Tángara Azulada & 1.7 & 5.36479304 & \\
\hline Icterus galbula & Chichiltote Norteño & 1.6 & 4.49613385 & \\
\hline Tyrannus forficatus & Tijereta Rosada & 1.6 & 1.70895245 & \\
\hline Icterus spurius & Chichiltote Castaño & 1.2 & 0.80867171 & \\
\hline Total & & & $\mathbf{4 0 7 . 1 0 0 3 2 8}$ & \\
\hline
\end{tabular}

Los pastos con árboles sirven para dar sombra al ganado, sin embargo, estos pueden atraer aves utilizándolos para percha, sombra o como corredor al desplazarse entre áreas abiertas, pudiendo incrementar el peligro de colisión con torres eólicas. Los pastizales sin árboles presentan el hábitat más predominante en la zona producto de una ganadería extensiva. Las especies con mayores valores de vulnerabilidad son el Pato Real (IVA=6), seguido del Rabiorcado Magno (IVA= 5.7) y Águila Pescadora (IVA= 5).

Los pastizales sin árboles son también una zona importante pues es aquí donde se observó la mayor concentración de golondrinas de Avión Zapador y Golondrina Común (IVA=4) en cantidades superiores a los 20,000 individuos. El valor promedio del IVA para este hábitat es de 3.4 y la mediana es de 3.1 (Tabla 6).

Tabla 6.- Valores IVA y MVP paras las especies de pastizales sin árboles. Eolo de Nicaragua S.A. 2011-2012.

\begin{tabular}{|l|l|c|c|c|}
\hline \multicolumn{1}{|c|}{ Nombre Científico } & $\begin{array}{c}\text { Nombre común en } \\
\text { español }\end{array}$ & IVA & IMVP total & IMVP med \\
\hline Cairina moschata & Pato Real & 6.0 & 6.59167373 & 6.59167373 \\
\hline Fregata magnificens & Rabihorcado Magno & 5.7 & 3.92783402 & 3.92783402 \\
\hline Pandion haliaetus & Águila Pescadora & 5.0 & 3.4657359 & 3.4657359 \\
\hline Cathartes aura & Zopilote Cabecirrojo & 4.7 & 6.54639004 & 6.54639004 \\
\hline Coragyps atratus & Zopilote Negro & 4.7 & 15.5636741 & 15.5636741 \\
\hline Parabuteo unicinctus & Gavilán Charreteado & 4.4 & 11.0440296 & 11.0440296 \\
\hline Quiscalus mexicanus & Zanate Grande & 4.4 & 24.0912667 & 24.0912667 \\
\hline Caracara cheriway & Caracara Crestado & 4.2 & 12.4822178 & 12.4822178 \\
\hline Hirundo rustica & Golondrina Común & 4.0 & 37.0418519 & 37.0418519 \\
\hline Riparia riparia & Avión Zapador & 4.0 & 38.4760015 & 38.4760015 \\
\hline
\end{tabular}

El Higo Revista Científica / Volumen 11. No. 01, pp. 69-89/ junio 2021 
José M. Zolotoff $P$.

\begin{tabular}{|l|l|c|c|c|}
\hline \multicolumn{1}{|c|}{ Nombre Científico } & \multicolumn{1}{|c|}{$\begin{array}{c}\text { Nombre común en } \\
\text { español }\end{array}$} & IVA & IMVP total & IMVP med \\
\hline Ardea alba & Garzón Grande & 3.9 & 2.69557237 & 2.69557237 \\
\hline Eupsittula canicularis & Perico Frentinaranja & 3.3 & 13.0400767 & 13.0400767 \\
\hline Bubulcus ibis & Garcilla Bueyera & 3.3 & 16.3755163 & 16.3755163 \\
\hline Patagioenas flavirostris & Paloma Piquirroja & 3.3 & 3.66204096 & 3.66204096 \\
\hline Zenaida asiatica & Tórtola Aliblanca & 3.3 & 2.3104906 & 2.3104906 \\
\hline Myiozetetes similis & Güis Chico & 3.1 & 4.91772695 & 197.314372 \\
\hline Agelaius phoeniceus & Tordo Sargento & 2.8 & 1.92540883 & \\
\hline Columbina inca & Tortolita Colilarga & 2.8 & 1.92540883 & \\
\hline Columbina talpacoti & Tortolita Rojiza & 2.8 & 5.40530597 & \\
\hline Egretta caerulea & Garceta Azul & 2.8 & 3.0517008 & \\
\hline Melanerpes hoffmannii & Carpintero Nuquigualdo & 2.8 & 3.0517008 & \\
\hline Sturnella magna & Zacatero Común & 2.8 & 9.25612364 & \\
\hline Crotophaga sulcirostris & Garrapatero Común & 2.4 & 8.23116758 & \\
\hline Setophaga petechia & Reinita Amarilla & 2.3 & 4.18077209 & \\
\hline Calocitta formosa & Urraca Copetona & 2.2 & 7.5582164 & \\
\hline Campylorhynchus rufinucha & Saltapiñuela Nuquirrufa & 2.2 & 3.08065414 & \\
\hline Egretta thula & Garceta Patiamarilla & 2.2 & 3.98168771 & \\
\hline Pitangus sulphuratus & Güis Común & 2.2 & 4.6209812 & \\
\hline Falco sparverius & Cernícalo Americano & 1.7 & 1.1552453 & \\
\hline Total & & 259.656472 & \\
\hline
\end{tabular}

En general, los valores más altos de vulnerabilidad en los cuatros tipos de hábitat se encuentran en las especies: Rabihorcado Magno, el Zopilote Negro, el Zopilote Cabecirroja, Águila Pescadora, Caracara Crestado, Zanate Nicaragüense, y la Garza Grande.

Con los valores de IVA calculado e IMVP total e IMVP medio para cada hábitat se procedió a calcular el percentil para ambos valores. La Tabla 7 presenta los resultados de los cálculos de percentiles para<P50 riesgo bajo, y >P50 riego alto tanto para MVP total y Media. 
José M. Zolotoff $P$.

Tabla 7.- Resultados de cálculos de percentiles para <P50 y >P50. Eolo de Nicaragua S.A. 2011-2012.

\begin{tabular}{|l|c|c|c|c|}
\hline & \multicolumn{2}{|c|}{ Riesgo Bajo } & \multicolumn{2}{c|}{ Riesgo Alto } \\
\hline MPV total & 1 & 2 & 3 & 4 \\
\hline $\mathrm{P}<50, \mathrm{P}>50$ & 259.65 & 310.57 & 368.49 & 407.1 \\
\hline & & 2da posición & 3ra posición & \\
\hline MVP med & 1 & 2 & 3 & 4 \\
\hline$P<50, P>50$ & 197.31 & 220.76 & 270.1 & 313.01 \\
\hline & & 2da posición & 3ra posición & \\
\hline
\end{tabular}

Obtenidos los rangos en los cuales el riesgo será Bajo o Alto, la Tabla 8 muestra el total de las sumatorias del MVP total y Medio calculado para cada hábitat, donde podemos determinar que el bosque ripario y pastizales con árboles son los sitios con mayor riesgo de colisión para instalar torres eólicas.

Tabla 8.- Valores para el Mapa de Vulnerabilidad Potencial para cada hábitat y tipo de riesgo. Eolo de Nicaragua S.A. 2011-2012.

\begin{tabular}{|l|c|c|c|}
\hline \multicolumn{1}{|c|}{ Hábitat } & MVP total & MVP med & Riesgo MVP Med \\
\hline Bosque Ripario & 368.49 & 270.1 & Alto \\
\hline Costa del lago & 310.57 & 220.76 & Bajo \\
\hline Pastizales con árboles & 407.1 & 313.01 & Alto \\
\hline Pastizales sin árboles & 259.65 & 197.31 & Bajo \\
\hline
\end{tabular}

Si bien el bosque ripario no se presenta como sitio para la instalación de torres eólicas, resulta interesante observar que de haberlo sido hubiese afectado el hábitat con mayor diversidad de especies, comprobando la efectividad del presente método.

La Figura 2 presenta el mapa de riesgos por tipo de hábitat ubicando las torres los cuales fueron instaladas en 2014. 


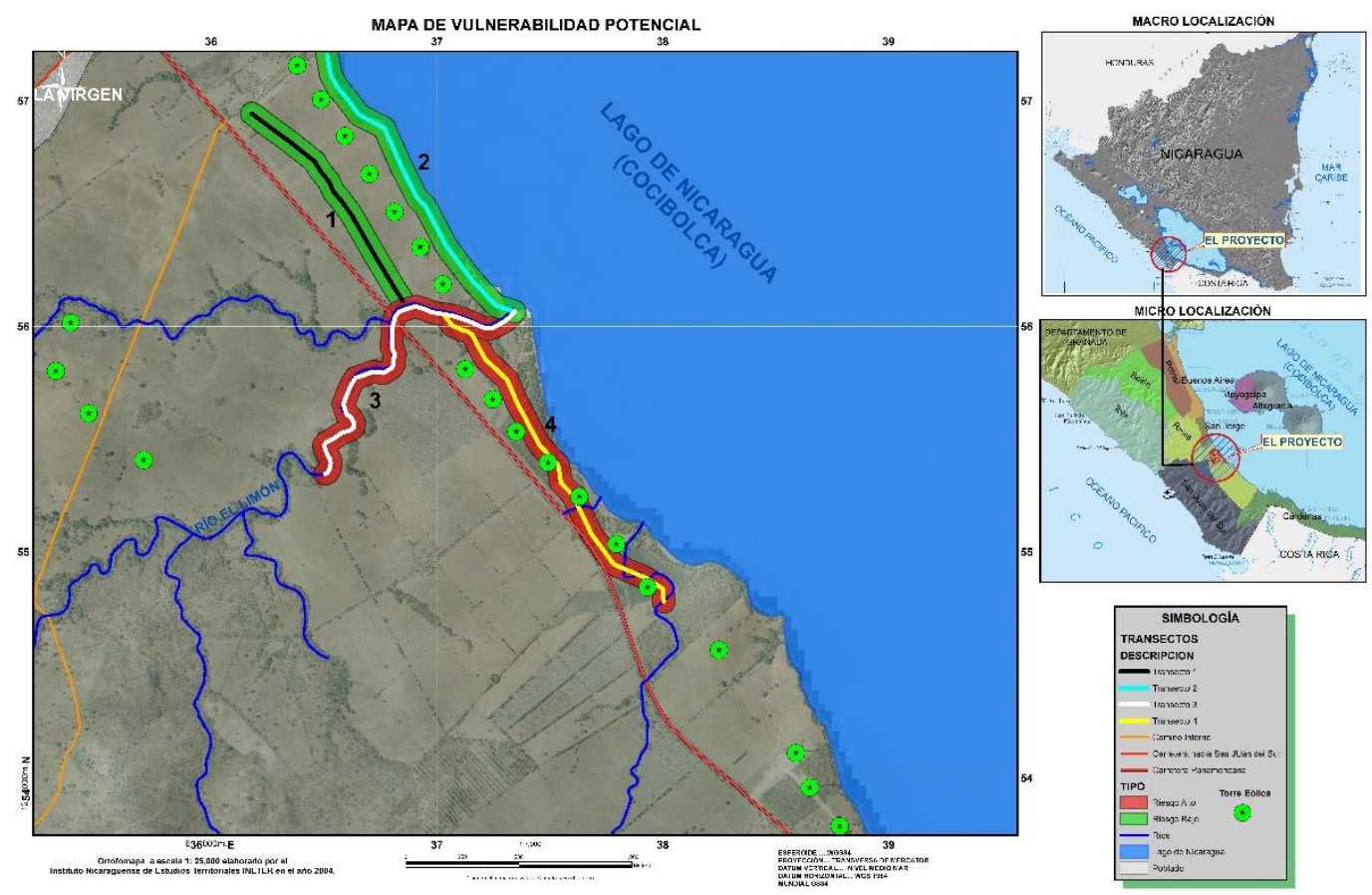

Figura 2.- Mapa de Vulnerabilidad Potencial. Eolo de Nicaragua S.A. 2011-2012.

Otra forma en la que se comprobó el método, es que posterior al presente estudio se continuó trabajando en el protocolo de colisión durante la etapa de operación, comprobando que la mayoría de las colisiones se registraban en las torres ubicadas en la zona de pastizales con árboles (observación personal).

\section{CONCLUSIONES}

El Índice de Vulnerabilidad de Aves y Mapa de Vulnerabilidad Potencial constituyen herramientas importantes que permiten identificar los riesgos potenciales de colisión de aves en centrales eólicas antes de su construcción.

Dichas herramientas deben de formar parte de los estudios de Evaluación de Impacto Ambiental en la etapa de pre construcción, resultando indispensable que toda empresa eólica en Nicaragua cuente con un protocolo de colisión durante la etapa de operación.

\section{REFERENCIAS}

American Wind Wildlife Institute (AWWI). (2016). Wind turbine interactions with wildlife and their habitats: a summary of research results and priority questions. Washington, DC. USA. Recuperado de https://awwi.org/resources/summary-of-wind-wildlife-interactions-2/. 
José M. Zolotoff $P$.

Arnett, EB., Brown, WK., Erickson, WP., Fiedler, JK., Hamilton BI., Henry, TH.,... Tankersley. Jr. RD. (2008). Patterns of bat fatalities at wind energy facilities in North America. The Journal of Wildlife Management, 72(1), 61-78.

Cornell Lab of Ornithology. (2015, 1 de mayo). Bird Guide. Recuperado de https://www.birds.cornell.edu/home/about/

Hernández-Sampieri, R., Collado, C., y Baptista, P. (2014). Metodología de la investigación. México, D.F., México: Mc Graw-Hill.

International Union for Conservation of Nature. (2016). Red list of threatened species. Recuperado de https://www.iucnredlist.org/

Manzanarez, R., Tórrez, M., Gutiérrez, A., Manzanares, J., y Gutiérrez, Z. (Ed.). (2018). Lista Roja de Especies Vertebradas en Riesgo de Extinción de Nicaragua. Managua, Nicaragua: Jóvenes Ambientalistas.

Martínez-Sánchez, J. C., Durioux, L. \& Muñoz, F. (2014). A guide to the birds of Nicaragua. Alemania: Westarp Science Fachvlge.

McCrary, J. \& Young, D. (2008). New and noteworthy observations of raptors in southward migration in Nicaragua. Ornitología Neotropical, 19(4), 573-580. Recuperado de https://www.researchgate.net/publication/228493827 New and noteworthy observation s of raptors in southward migration in Nicaragua

Pérez, A. M. (2004). Aspectos Conceptuales, Análisis numérico, Monitoreo y Publicación de Datos sobre Biodiversidad. Managua, Nicaragua: MARENA - ARAUCARIA.

Ralph, C.J., Geupel, G., Pyle, P., Martin, T., DeSante, D. y Milá, B. (1996). Manual de métodos de campo para el monitoreo de aves terrestres. Albany, CA: Pacific Southwest Research Station.

Recuperado

de https://www.fs.fed.us/psw/publications/documents/psw gtr159/psw gtr159.pdf

Stiles, G. \& Skutch, A. (1989). A guide to the birds of Costa Rica. New York, USA: Cornell University Press.

Strickland, M.D., Arnett, E.B., Erickson, W.P., Johnson, D.H., Johnson, G.D., Morrison, M.L., Shaffer, J.A., \& Warren-Hicks, W. (2011). Comprehensive guide to studying wind energy/wildlife interactions. Washington, DC: The National Wind Coordinating Collaborative.

Wunderle, J. (1994). Métodos de campo para contar aves terrestres del Caribe. USDA Forest Service, Souther Forest Experiment Station. Recuperado de

El Higo Revista Científica / Volumen 11. No. 01, pp. 69-89/ junio 2021 
https://www.researchgate.net/publication/283349293 Metodos Para Contar Aves Terres tres Del Caribe/link/5636168908aeb786b703d6e8/download

\section{SEMBLANZA DEL AUTOR}



José M. Zolotoff-Pallais. Licenciado en Ecología y Recursos Naturales. Diplomado en Evaluación de Impacto Ambiental, Economía de RN, Gestión Ambiental, y Servicios Ambientales. Con 22 años de experiencia en ornitología a nivel nacional. Siete años de experiencia en monitoreo de fauna en centrales eólicas en Nicaragua y otros países en Centroamérica. Ha dictado múltiples talleres sobre identificación y conservación de aves en Nicaragua. Director del Área de Investigación y Capacitación de Fundación Cocibolca, para la Reserva Natural Volcán Mombacho desde 1996, Granada. Es autor de varios artículos científicos sobre conservación de aves entre estos Areas Importantes para Aves en Nicaragua, Estado de Conservación de Aves Acuáticas y sus Habitas en Nicaragua, Areas Claves de Biodiversidad en Nicaragua, así como co autor de la lista roja de especies de aves en peligro en Nicaragua. josezolotoff@gmail.com 